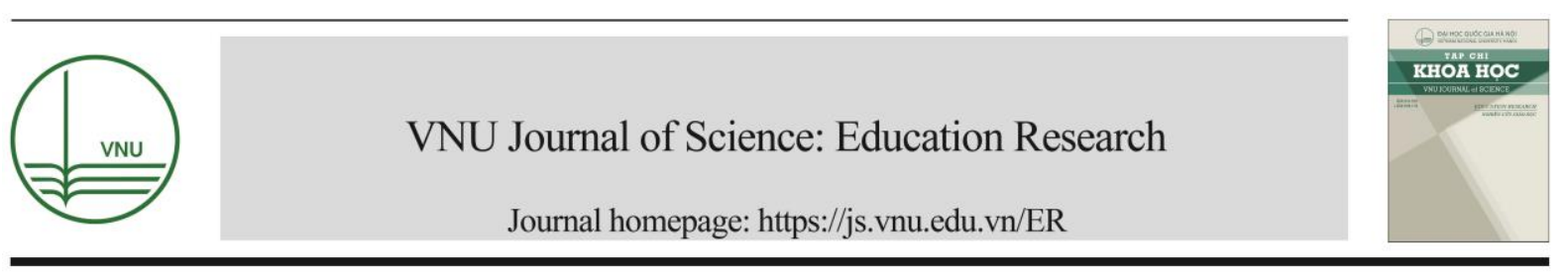

Original Article

\title{
Developing a Toolkit for Measuring the Levels of Education Quality Assurance of Higher Education Institutions in Vietnam: Problems and Solutions
}

\author{
Ta Thi Thu Hien ${ }^{1, *}$, Nguyen Thi Thu Huong ${ }^{1}$, Tran Huu Luong ${ }^{1}$, \\ Nguyen Huu Cuong ${ }^{2}$, Bui Thi Thu Thuy ${ }^{1}$, Nguyen Hoa Huy ${ }^{1}$, Trinh Thi Nhung ${ }^{1}$, \\ Nguyen Thi Kim Anh ${ }^{1}$, Vu Minh Phuong ${ }^{1}$, \\ ${ }^{1}$ VNU Centre for Education Accreditation, 144 Xuan Thuy, Cau Giay, Hanoi, Vietnam \\ ${ }^{2}$ Ton Duc Thang University, 19 Nguyen Huu Tho, District 7, Ho Chi Minh City, Vietnam
}

Received 21 October 2020

Revised 27 October 2020; Accepted 27 October 2020

\begin{abstract}
This paper discusses the issues relating to the toolkit for measuring the quality assurance level of higher education institutions, the process of developing and detailing the toolkit for its usage in the self-assessment and external assessment process in compliance with the quality assessment standards for higher education institutions issued by the Ministry of Education and Training. Therefrom, the paper proposes solutions for improving the implementation of the toolkit in quality assurance practices within higher education institutions and supporting assessors, accreditation bodies and government agencies for quality evaluation, monitoring and management.

Keywords: Quality assessment standards, higher education institution, toolkit, higher education quality assurance, education quality accreditation.
\end{abstract}

\footnotetext{
${ }^{*}$ Corresponding author.

E-mail address: tahien@vnu.edu.vn

https://doi.org/10.25073/2588-1159/vnuer.4478
} 


\title{
Xây dựng Bộ công cụ đo lường mức độ đảm bảo chất lượng của các cơ sở giáo dục đại học ở Việt Nam: Vấn đề và giải pháp
}

\author{
Tạ Thị Thu Hiền ${ }^{1, *}$, Nguyễn Thị Thu Hương${ }^{1}$, Trần Hữu Lượng ${ }^{1}$, \\ Nguyễn Hữu Cương ${ }^{2}$, Bùi Thị Thu Thủy ${ }^{1}$, Nguyễn Hoà Huy ${ }^{1}$, \\ Trịnh Thị Nhung ${ }^{1}$, Nguyễn Thị Kim Ánh ${ }^{1}$, Vũ Minh Phương ${ }^{1}$ \\ ${ }^{1}$ Trung tâm Kiểm định chất lượng giáo dục, Đại học Quốc gia Hà Nội, \\ 144 Xuân Thủy, Cầu Gián, Hà Nội, Việt Nam \\ ${ }^{2}$ Truờng Đại học Tôn Đức Thắng, 19 Nguyễn Hũu Tho, \\ Quận 7, Thành phố Hồ Chí Minh, Việt Nam \\ Nhận ngày 21 tháng 10 năm 2020 \\ Chỉnh sửa ngày 27 tháng 10 năm 2020; Chấp nhận đăng ngày 27 tháng 10 năm 2020
}

\begin{abstract}
Tóm tắt: Đảm bảo và kiểm định chất lượng giáo dục là công cụ quản lý chất lượng quan trọng giúp các cơ sở giáo dục đại học, cơ quan quản lý Nhà nước và xã hội quản lý, giám sát chất lượng giáo dục đại học. Trong việc tồ chức hoạt động đảm bảo và kiểm định chất lượng cơ sở giáo dục đại học ở Việt Nam, việc thống nhất quan điểm để đo lường mức độ đảm bảo chất lượng của cơ sở giáo dục đại học nhằm đưa ra các nhận định phù hợp đối với chất lượng của cơ sở giáo dục đại học là vấn đề không chỉ các cơ sở giáo dục đại học mà các tổ chức kiểm định chất lượng giáo dục đặc biệt quan tâm. Bài viết này trình bày các vấn đề về công cụ đo lường mức độ đảm bảo chất lượng của cơ sở giáo dục đại học, quy trình xây dựng và chi tiết hoá công cụ đo lường mức độ đảm bảo chất lượng của cơ sở giáo dục đại học để sử dụng trong quá trình tự đánh giá, quá trình đánh giá ngoài theo Bộ tiêu chuẩn đánh giá chất lượng cơ sở giáo dục đại học do Bộ Giáo dục và Đào tạo ban hành. Đồng thời, các tác giả cũng đề xuất các giải pháp sử dụng Bộ công cụ trong các hoạt động đảm bảo chất lượng bên trong cơ sở giáo dục đại học và hỗ trợ các chuyên gia đánh giá ngoài, các tổ chức kiểm định chất lượng giáo dục và cơ quan quản lý nhà nước thực hiện đánh giá, giám sát và quản lý chất lượng.
\end{abstract}

Tù khóa: Tiêu chuẩn đánh giá chất lượng, cơ sở giáo dục đại học, công cụ, đảm bảo chất lượng giáo dục đại học, kiểm định chất lượng giáo dục.

\section{1. Đặt vấn đề}

Hệ thống đảm bảo chất lượng (ĐBCL) giáo dục đại học đã được quy định trong Luật sửa đổi, bổ sung một số điều của Luật Giáo dục đại học, bao gồm hệ thống bảo đảm chất lượng bên trong và hệ thống bảo đảm chất lượng bên ngoài. Kiểm định chất lượng giáo dục

\footnotetext{
* Tác giả liên hệ.

Địa chi email: tahien@vnu.edu.vn

https://doi.org/10.25073/2588-1159/vnuer.4478
}

(KĐCLGD) được coi là cơ chế ĐBCL từ bên ngoài hiệu quả, giúp các cơ sở giáo dục đại học (CSGDĐH) giải trình chất lượng với xã hội, đồng thời để xã hội biết và giám sát chất lượng giáo dục [1]. Thực thi các điều khoản của Luật, Bộ Giáo dục và Đào tạo (GDĐT) đã ban hành tiêu chuẩn đánh giá chất lượng, quy trình và chu kỳ KĐCLGD đại học, đồng thời chủ trương cập nhật sớm các tiêu chuẩn quốc tế trong các hoạt động KĐCLGD, đẩy mạnh KĐCLGD đối với CSGDĐH theo hướng tiếp cận chuẩn khu vực và quốc tế. Theo đó, ngày 19/5/2017, Bộ 
trưởng Bộ GDĐT đã ban hành Thông tư số 12/2017/TT-BGDĐT quy định về kiểm định chất lượng CSGDĐH (sau đây gọi là Thông tư 12), trong đó quy định về bộ tiêu chuẩn đánh giá chất lượng CSGDĐH. Bộ tiêu chuẩn này gồm 25 tiêu chuẩn, 111 tiêu chí; các tiêu chuẩn được phân theo 4 nhóm: ĐBCL về chiến lược (8 tiêu chuẩn, 37 tiêu chí); ĐBCL về hệ thống (4 tiêu chuẩn, 19 tiêu chí); ĐBCL về thực hiện chức năng (9 tiêu chuẩn, 39 tiêu chí) và Kết quả hoạt động (4 tiêu chuẩn, 16 tiêu chí) [2]. Bộ tiêu chuẩn này được xây dựng trên nền tảng Bộ tiêu chuẩn đánh giá ĐBCL cơ sở giáo dục của Mạng lưới ĐBCL các trường đại học ASEAN (AUN-QA), với những tiếp cận mới trong quản trị đại học. Tiếp đó, Cục Quản lý chất lượng, Bộ GDĐT đã ban hành các văn bản hướng dẫn thực hiện tự đánh giá và đánh giá ngoài [3-6]. Tính đến tháng 9/2020 đã có 28 CSGDĐH được KĐCLGD theo bộ tiêu chuẩn mới ban hành theo Thông tư 12 này [7]. Trong quá trình thực hiện của các CSGDĐH và tổ chức KĐCLGD, nhiều vấn đề đã được đặt ra và cần có sự thống nhất về quan điểm tiếp cận bộ tiêu chuẩn, phương thức, công cụ đánh giá mức độ ĐBCL của cơ sở giáo dục. Do đó, việc xây dựng một bộ công cụ chi tiết để đo lường mức độ ĐBCL của CSGDĐH là hết sức cần thiết.

Bài báo này sẽ trình bày quy trình nghiên cứu xây dựng và cụ thể hoá Bộ công cụ đo lường mức độ ĐBCL của CSGDĐH (sau đây gọi là Bộ công cụ) để sử dụng trong quá trình tự đánh giá, đánh giá ngoài theo Thông tư 12; đồng thời đề xuất các giải pháp sử dụng Bộ công cụ trong các hoạt động ĐBCL bên trong CSGDĐH, hỗ trợ các chuyên gia đánh giá ngoài, các tổ chức KĐCLGD và cơ quan quản lý Nhà nước trong triển khai thực hiện đánh giá, giám sát và quản lý chất lượng giáo dục.

\section{Phương pháp nghiên cứu}

Nghiên cứu sử dụng kết hợp các phương pháp định lượng và định tính, trong đó tập trung nghiên cứu tài liệu, khảo sát bằng phiếu hỏi, phỏng vấn sâu, tham vấn chuyên gia về các vấn đề liên quan đến Bộ tiêu chuẩn theo Thông tư 12 , các văn bản hướng dẫn thực hiện của Bộ GDĐT và bản dự thảo Bộ công cụ do nhóm nghiên cứu xây dựng. Các phiếu khảo sát được xây dựng theo quy trình gồm 6 bước: i) Xác định mục đích phiếu hỏi; ii) Xác định các tiêu chí đánh giá; iii) Xây dựng các chỉ báo tương ứng; iv) Dự thảo phiếu hỏi; v) Xin ý kiến chuyên gia; và vi) Điều chỉnh và hoàn thiện phiếu hỏi để tiến hành khảo sát. Thang đánh giá sử dụng được thiết kế theo thang Likert 5 mức độ; được kiểm tra độ tin cậy thông qua hệ số Cronbach's Alpha. Nội dung các phiếu khảo sát được thiết kế để phù hợp với các đối tượng cần khảo sát, bao gồm: Phiếu [M01] gồm 02 câu hỏi chính, trong đó có 6 biến đánh giá về Bộ tiêu chuẩn đánh giá chất lượng $\mathrm{CSGDĐH} \mathrm{(theo}$ Thông tư 12), các văn bản hướng dẫn thực hiện Bộ tiêu chuẩn do Bộ GDĐT ban hành và 9 biến đánh giá mức độ cần thiết của các đề xuất bổ sung đối với văn bản hướng dẫn thực hiện Bộ tiêu chuẩn để sử dụng hiệu quả hơn trong quá trình đánh giá. Phiếu [M02] gồm 03 câu hỏi chính, trong đó có 13 biến đánh giá về cấu trúc và các nội dung, 5 biến đánh giá tính khả thi và 01 câu hỏi mở lấy ý kiến góp ý để hoàn thiện Bộ công cụ đo lường mức độ $\mathrm{DBCL}$ của CSGDĐH. Ngoài ra, các vấn đề được trao đổi, bàn luận trong phỏng vấn, thảo luận nhóm cũng tập trung về những mặt hạn chế và đề xuất cải tiến để tăng hiệu quả sử dụng Bộ tiêu chuẩn đánh giá chất lượng $\mathrm{CSGDĐH} \mathrm{và} \mathrm{các} \mathrm{văn} \mathrm{bản}$ hướng dẫn của Bộ GDĐT trong quá trình đánh giá chất lượng.

Nghiên cứu sử dụng phương pháp chọn mẫu thuận tiện kết hợp phân tầng đối với 125 người là cán bộ quản lý các cấp, chuyên gia thuộc tổ chức KĐCLGD, cán bộ quản lý và cán bộ ĐBCL giáo dục các CSGDĐH. Hình thức khảo sát được thực hiện online kết hợp phát phiếu trực tiếp; mỗi người chỉ trả lời một lần và yêu cầu bắt buộc đối với tất cả câu hỏi trong phiếu. Nhóm nghiên cứu đã nhận được 104 phiếu hợp lệ, trong đó có $11,5 \%$ là chuyên gia đánh giá của các tổ chức KĐCLGD và $37,5 \%$ là 
các cán bộ ĐBCL của CSGDĐH, đồng thời có $88,5 \%$ người đã hoàn thành và có chứng chỉ hoàn thành chương trình đào tạo kiểm định viên, 43,3\% đã có thẻ kiểm định viên; ngoài ra, còn có 5 cán bộ cơ quan quản lý nhà nước về KĐCLGD và 20 người là cán bộ quản lý CSGDĐH, tổ chức KĐCLGD. Dữ liệu khảo sát được tập hợp, làm sạch và xử lý bằng các phần mềm Excel và SPSS 25; kết quả khảo sát được phân tích, đánh giá làm căn cứ để hoàn thiện Bộ công cụ và đưa ra các kiến nghị, giải pháp sử dụng. Ngoài ra, nhóm tác giả kết hợp nghiên cứu tài liệu, tham vấn ý kiến 02 chuyên gia và phỏng vấn sâu 10 kiểm định viên, đánh giá viên, cán bộ quản lý, cán bộ ĐBCL ở các CSGDĐH. Thông tin từ phỏng vấn, tham vấn chuyên gia được sử dụng để bổ trợ cho các đánh giá định lượng từ khảo sát.

\section{Kết quả nghiên cứu}

3.1. Cơ sở đề xuất xây dưng Bộ công cu đo luờng múc độ đảm bảo chất lương của co sở giáo dục đại học ở Việt Nam

3.1.1. Chủ trương, các quan điểm tiếp cận về đảm bảo và kiểm định chất lượng $\mathrm{CSGDĐH}$ ở Việt Nam

Hệ thống đảm bảo, KĐCLGD ở Việt Nam được hình thành và phát triển gần 20 năm, bắt nguồn từ các chủ trương của Nhà nước, Chính phủ (từ 2001) [8] và quy định của Bộ GDĐT (từ 2004) [9]. KĐCLGD được xác định là một trong những giải pháp quan trọng để quản lý, nâng cao chất lượng giáo dục, được thể hiện trong các Nghị quyết của Đảng, đã chính thức được luật hóa với đầy đủ các thể chế trong Luật Giáo dục và Luật Giáo dục đại học. Nghị quyết số 29-NQ/TW Hội nghị lần thứ 8 Ban chấp hành Trung ương khóa XI về Đổi mới căn bản, toàn diện GDĐT đã đề ra giải pháp "Hoàn thiện hệ thống kiểm định chất lượng giáo duc. Định kỳ kiểm định chất lượng các co sở giáo duc đào tạo và các chuoong trình đào tạo; công khai kết quả kiểm định" [10].
Theo các quy định của Luật Giáo dục đại học và Luật sửa đổi, bổ sung một số điều của Luật Giáo dục đại học, KĐCLGD là bắt buộc và được thực hiện định kỳ đối với cơ sở giáo dục và chương trình đào tạo $[1,11]$ và hướng đến các mục tiêu: i) bảo đảm và nâng cao chất lượng giáo dục đại học; ii) xác nhận mức độ đáp ứng mục tiêu của CSGDĐH hoặc chương trình đào tạo trong từng giai đoạn; iii) làm căn cứ để CSGDĐH giải trình với chủ sở hữu, cơ quan có thẩm quyền, các bên liên quan và xã hội về thực trạng chất lượng đào tạo và iv) làm cơ sở cho người học lựa chọn CSGDĐH, chương trình đào tạo; cho nhà tuyển dụng lao động tuyển chọn nhân lực [1]. Theo đó, thực hiện chức năng quản lý Nhà nước về KĐCLGD, Bộ GDĐT đã ban hành hệ thống các văn bản quản lý để triển khai hoạt động đảm bảo và KĐCLGD. Đồng thời, liên tục trong các năm từ 2018 đến nay, Bộ GDĐT đã xác định mục tiêu xuyên suốt trong 9 nhóm nhiệm vụ chủ yếu các năm học 2018-2019, 2019-2020 và 2020-2021 là tiếp tục đổi mới và ĐBCL giáo dục; tăng cường công tác khảo thí, đánh giá. KĐCLGD được xác định là một trong 5 nhóm giải pháp cơ bản của ngành, trong đó nhiệm vụ được nêu rõ là tập trung kiểm định chất lượng các cơ sở giáo dục và chương trình đào tạo (với các chỉ tiêu cụ thể theo từng giai đoạn) để hướng tới cải tiến chất lượng, từng bước hình thành và xây dựng văn hóa chất lượng nhà trường [12]. Sau gần 20 năm hình thành và phát triển, đảm bảo và KĐCLGD đã được ghi nhận là một giải pháp quan trọng để quản lý, nâng cao chất lượng giáo dục.

Mặc dù đi sau các nước có hệ thống đảm bảo, KĐCLGD phát triển như Châu Âu, Hoa Kỳ, Úc,... giáo dục đại học Việt Nam đã kế thừa các quan điểm tiếp cận đã được kiểm nghiệm và triển khai thực tế thành công. Từ các quan điểm và mô hình chất lượng của các tổ chức đảm bảo, KĐCL như INQAAHE, TESQA, QAA, APQN, AQAN, AUN-QA,... và một số quan điểm tiếp cận về đảm bảo, kiểm định chất lượng CSGDĐH ở các nước phát triển đã được sử dụng làm căn cứ cho việc xây 
dựng hệ thống cũng như triển khai các hoạt động đảm bảo, KĐCLGD của Việt Nam. Trong đó, có thể kể đến quan điểm của AUN-QA "đánh giá đảm bảo chất luợng co sở giáo duc trong giáo duc đại hoc được xem nhu đánh giá một hệ thống chiến luợc, chiến thuật và chức năng đảm bảo chất luợng trong đào tạo, nghiên cưu khoa hoc và phuc vu cộng đồng cũng nhu các kết quả và hiệu quả của hệ thống đảm bảo chất luợng của tổ chức. Đánh giá đảm bảo chất luợng co sở giáo duc nhằm muc đỉch xác dịnh xem cơ sở giáo dục đã đáp úng các tiêu chuẩn chất luơng đã đurợc quy định và các yêu cầu nhu thế nào" [13]. AUN-QA tiếp cận việc đánh giá ĐBCL CSGDĐH theo phương pháp đánh giá theo nguyên lý (priciple-based assessment), với quan điểm tiếp cận hệ thống, chất lượng có tính gắn kết và đảm bảo tính hệ thống, với trọng tâm là cải tiến chất lượng liên tục, đáp ứng nhu cầu và sự hài lòng của các bên liên quan trong cả quá trình thực hiện. Theo quan điểm này, việc đánh giá được thực hiện bởi những chuyên gia đồng cấp có kỹ năng, tiến hành tham chiếu theo tiêu chí và không áp đặt ý kiến chủ quan; đánh giá để xác thực thông tin, minh chứng đáp ứng các tiêu chuẩn của AUN-QA với hai nguyên tắc cơ bản là: độc lập (là cơ sở cho tính khách quan, minh bạch của các kết luận đánh giá) và minh chứng (là cơ sở lập luận để đạt được các kết luận đánh giá đáng tin cậy và có khả năng sử dụng lại trong quy trình đánh giá hệ thống) [13].

Kế thừa bộ tiêu chuẩn đánh giá $\mathrm{DBCL}$ CSGDĐH của AUN-QA, Bộ GDĐT đã ban hành Bộ tiêu chuẩn đánh giá chất lượng CSGDĐH tại Thông tư 12 và phát triển phương pháp đánh giá theo quy định (rule-based assessment) kết hợp với đánh giá theo nguyên lý. Thông tư 12 quy định rõ về quy trình, chu kỳ kiểm định chất lượng CSGDĐH và tiêu chuẩn đánh giá chất lượng gồm 25 tiêu chuẩn, 111 tiêu chí bao quát bốn lĩnh vực (ĐBCL về chiến lược, ĐBCL về hệ thống, ĐBCL về thực hiện chức năng và Kết quả thực hiện); mỗi tiêu chí được đánh giá theo thang 7 mức (tương ứng với 7 điểm) [4]. Điểm của mỗi tiêu chí được đánh giá theo điểm nguyên (từ 1-7); điểm của mỗi tiêu chuẩn là điểm trung bình cộng điểm của các tiêu chí trong tiêu chuẩn (làm tròn đến 2 chữ số thập phân); điểm trung bình của các tiêu chuẩn trong mỗi muc (4 lĩnh vực ĐBCL) là điểm trung bình cộng của các tiêu chuẩn trong mục (làm tròn đến 2 chữ số thập phân). Một trong các điều kiện để CSGDĐH được công nhận đạt tiêu chuẩn chất lượng giáo dục là CSGDĐH đó đạt điểm trung bình của các tiêu chuẩn trong từng mục từ 3,5 điểm trở lên và không tiêu chuẩn nào có điểm trung bình dưới 2,0 điểm. Để thực hiện, Cục Quản lý chất lượng, Bộ GDĐT đã ban hành các văn bản hướng dẫn cụ thể về tự đánh giá (Công văn số 766/QLCL-KĐCLGD ngày 20/4/2018) dành cho các CSGDĐH; về đánh giá ngoài (Công văn số 767/QLCL-KĐCLGD ngày 20/4/2018) dành cho các chuyên gia đánh giá và tổ chức KĐCLGD; hướng dẫn đánh giá ngoài theo bộ tiêu chuẩn đánh giá chất lượng $\mathrm{CSGDĐH}$ (Công văn số 768/QLCL-KĐCLGD ngày 20/4/2018, sau đó được thay thế bởi Công văn 1668/QLCL-KĐCLGD ngày 31/12/2019) dùng chung cho các đối tượng để triển khai tự đánh giá và đánh giá ngoài. Công văn 768 và 1668 đã hướng dẫn cụ thể hơn về cấu trúc của bộ tiêu chuẩn đánh giá chất lượng CSGDĐH và giải thích rõ hơn về thang đánh giá 7 mức với hai yêu cầu (tương ứng với hai thang đo) về mức độ đáp ứng về hoạt động ĐBCL và mức độ đáp ứng về minh chứng; đồng thời hướng dẫn đánh giá theo chu trình PDCA (Plan-Do-Check-Act) và cung cấp bảng hướng dẫn đánh giá để cụ thể hóa các yêu cầu của từng tiêu chí, hướng dẫn các mốc chuẩn tham chiếu để xem xét mức độ đạt yêu cầu tối thiểu và gợi ý những nguồn minh chứng có thể khai thác. Các yêu cầu, hướng dẫn này đã được các CSGDĐH, các tổ chức KĐCLGD sử dụng trong đánh giá thực tế 28 CSGDĐH tính đến thời điểm 30/9/2020 [7].

3.1.2. Yêu cầu từ thực tiễn sử dụng Bộ công cụ trong đánh giá, kiểm định chất lượng CSGDĐH ở Việt Nam

Hệ thống KĐCLGD đại học ở Việt Nam có thể phân chia thành 03 đối tượng chính, có quan 
hệ phụ thuộc lẫn nhau bao gồm hệ thống quản lý Nhà nước trong đó có các văn bản pháp lý về KĐCLGD do Nhà nước và Bộ GDĐT ban hành; hệ thống tổ chức KĐCLGD và đội ngũ nhân sự thực hiện KĐCLGD. Việt Nam đã xây dựng được hệ thống các văn bản pháp lý bao quát các lĩnh vực trong hoạt động đảm bảo và KĐCLGD đại học nói chung, kiểm định chất lượng CSGDĐH nói riêng (bao gồm hệ thống văn bản từ luật, nghị định đến thông tư, quy định, hướng dẫn). Về nguồn lực thực hiện KĐCLGD đại học, hiện nay Việt Nam có 5 tổ chức KĐCLGD, trong đó 4 tổ chức do Bộ GDĐT quyết định thành lập (Trung tâm KĐCLGD - Đại học Quốc gia Hà Nội, Trung tâm KĐCLGD - Đại học Quốc gia Thành phố Hồ Chí Minh, Trung tâm KĐCLGD - Đại học Đà Nẵng và Trung tâm KĐCLGD - Trường Đại học Vinh) và 01 tổ chức do Bộ GDĐT cho phép thành lập (Trung tâm KĐCLGD trực thuộc Hiệp hội các trường đại học, cao đẳng Việt Nam) và khoảng 2000 người được đào tạo kiểm định viên KĐCLGD đại học và trung cấp chuyên nghiệp, trong đó có 346 người đã được Bộ GDĐT tuyển chọn cấp thẻ kiểm định viên. Tính đến hết tháng 9/2020, cả nước đã có 230/241 CSGDĐH hoàn thành báo cáo tự đánh giá, trong đó có 155 lượt CSGDĐH (152 CSGDĐH) đã được đánh giá ngoài (trong giai đoạn từ 20142020) và 145 CSGDĐH đã được cấp giấy chứng nhận KĐCLGD, trong số đó có 28 CSGDĐH được đánh giá theo bộ tiêu chuẩn ban hành theo Thông tư 12 [7]. Với tốc độ triển khai các hoạt động kiểm định chất lượng CSGDĐH như hiện tại, dự tính đến hết năm 2020, hoạt động KĐCLGD đại học của Việt Nam chưa đáp ứng được các chỉ tiêu theo kế hoạch của Bộ GDĐT là "Đến hết ngày 31/12/2020, tất cả các truờng đại học, truờng cao đẳng su phạm, trung cấp su phạm đủ điều kiện được kiểm định và công bố công khai các kết quả kiểm định" [14].

Qua quá trình nghiên cứu, tổng hợp thông tin, tiếp thu các chia sẻ kinh nghiệm từ các chuyên gia đánh giá ngoài và kết quả khảo sát, nhóm nghiên cứu nhận thấy thực trạng triển khai hoạt động kiểm định chất lượng CSGDĐH trong thời gian qua còn một số vấn đề tồn tại cần được quan tâm khắc phục như:

Một là, bộ tiêu chuẩn đánh giá chất lượng CSGDĐH ban hành tại Thông tư 12 được kế thừa từ bộ tiêu chuẩn đánh giá $\mathrm{DBCL}$ của AUN-QA nên có nhiều nội dung tiếp cận mới về quản trị đại học, quản trị chiến lược, quản trị hệ thống bao quát rộng trên mọi lĩnh vực hoạt động của $\mathrm{CSGDĐH, \ldots .} \mathrm{Thực} \mathrm{tiểंn} \mathrm{triển} \mathrm{khai} \mathrm{còn}$ gặp khó khăn, do có sự chưa thống nhất trong quan điểm, cách tiếp cận và phương pháp đánh giá giữa các chuyên gia đánh giá ngoài của tổ chức KĐCLGD cũng như tại các CSGDĐH trong quá trình triển khai tự đánh giá. Ngoài ra, Bộ tiêu chuẩn được AUN-QA công bố trong Hướng dẫn đánh giá cơ sở giáo dục phiên bản 2.0 tiếp cận theo quan điểm đánh giá $\mathrm{DBCL}$; mỗi tiêu chuẩn được xây dựng tiếp cận theo chu trình P-D-C-A (Plan-Do-Check-Act) nhằm nắm bắt thông tin, minh chứng để đưa ra nhận định CSGDĐH đã thực hiện ĐBCL như thế nào. Trong khi đó, Bộ tiêu chuẩn được Bộ GDĐT ban hành tại Thông tư 12 mặc dù được kế thừa từ Bộ tiêu chuẩn của AUN-QA, nhưng là công cụ để thực hiện tự đánh giá và kiểm định chất lượng CSGDĐH. Mặt khác, phương pháp tiếp cận chủ yếu trong đánh giá lại khác nhau: AUN-QA đánh giá theo nguyên lý, còn ở Việt Nam các chuyên gia đánh giá ngoài chủ yếu mới tiếp cận kiểm định chất lượng theo nguyên tắc/quy định.

Hai là, mặc dù hệ thống văn bản hướng dẫn việc thực hiện kiểm định chất lượng CSGDĐH đã được Bộ GDĐT ban hành khá đầy đủ, nhưng chưa thật sự hoàn thiện, đồng bộ để giúp các CSGDĐH triển khai ĐBCL bên trong cũng như các chuyên gia đánh giá và các tổ chức KĐCLGD thống nhất về quan điểm tiếp cận, cách thức thực hiện đánh giá. Phản hồi của các cán bộ làm công tác ĐBCL trong các CSGDĐH trong thực tiễn triển khai các hoạt động đánh giá cho thấy, các yêu cầu của tiêu chí và mốc chuẩn tham chiếu còn chưa thực sự đảm bảo tính tương thích và rõ ràng. Vì vậy trong quá trình tự đánh giá, các CSGDĐH còn khá lúng túng khi phải kết nối những vấn đề cụ thể trong 
thực trạng hoạt động của CSGDĐH với các nội dung trong bộ tiêu chuẩn đánh giá chất lượng. Các chuyên gia đánh giá trong quá trình đánh giá ngoài cũng gặp không ít khó khăn khi đưa ra quyết định về mức đánh giá cho từng tiêu chí bởi tính đa dạng trong các vấn đề đánh giá cũng như tính chất phức tạp trong yêu cầu của thang đánh giá mà các hướng dẫn thực hiện chưa thực sự đầy đủ để giúp giải quyết khó khăn này một cách hiệu quả. Các tổ chức KĐCLGD cũng phải chủ động nghiên cứu để xây dựng thêm hệ thống các văn bản quản lý, các biểu mẫu, hướng dẫn chi tiết riêng để triển khai hoạt động KĐCLGD cũng như hỗ trợ các chuyên gia đánh giá trong quá trình tác nghiệp.

Kết quả khảo sát của nhóm nghiên cứu cũng cho thấy: tỷ lệ người trả lời đồng ý ở mức rất cao với các nhận định: Bộ tiêu chuẩn đã đề cập đầy đủ các lĩnh vục hoạt động của CSGDĐH và Các tiêu chí thể hiện đầy đủ yêu cầu cần đáp ứng mỗi tiêu chuẩn theo tiếp cânn $P D C A$ (lần lượt là $94,2 \%$ và $85,6 \%$ tổng số người trả lời). Nhưng tỷ lệ người trả lời không đồng ý cũng khá cao đối với các nhận định: Các nguồn minh chưng gợi ý trong văn bản huớng dẫn phù hợp với thực tiễn; Các mốc chuẩn tham chiếu trong văn bản huớng dẫn đã cu thể hóa được nọi hàm các yêu cầu của tiêu chí và Yêu cầu của tiêu chí cu thể, rõ ràng (lần lượt là $36,5 \%, 31,7 \%$ và $27,9 \%$ tổng số phản hồi). Dữ liệu cũng cho thấy các đối tượng không đồng ý hoặc phân vân về các nội dung này chủ yếu là các cán bộ ĐBCL của CSGDĐH.

Ba là, đội ngũ cán bộ thực hiện KĐCLGD còn thiếu về số lượng và chưa đồng đều về chất lượng. Mặc dù số lượng kiểm định viên và đánh giá viên được đào tạo của cả nước hiện tại là khoảng 2000 người, nhưng chỉ có chưa đến $10 \%$ trong số đó đã trực tiếp tham gia hoạt động kiểm định chất lượng CSGDĐH. Để đưa ra "phán quyết" cho một vấn đề cụ thể của CSGDĐH, đòi hỏi các chuyên gia đánh giá không chỉ cần hiểu đúng, hiểu sâu về tiêu chuẩn, tiêu chí đánh giá mà còn cần có nhiều kinh nghiệm trong các lĩnh vực quản trị đại học, khoa học giáo dục, đảm bảo và KĐCLGD cũng như các vẩn đề khác liên quan trực tiếp đến các lĩnh vực hoạt động của CSGDĐH để có thể có những đánh giá khách quan, chính xác cũng như có những tư vấn phù hợp, hữu ích cho sự phát triển của CSGDĐH.

Các vấn đề tồn tại từ thực tế triển khai kiểm định chất lượng các CSGDĐH nói trên đặt ra yêu cầu về việc cần có cách thức, công cụ hướng dẫn cụ thể hơn cho các CSGDĐH, các chuyên gia đánh giá, các tổ chức KĐCLGD để triển khai hiệu quả hơn công tác tự đánh giá, KĐCLGD. Điều này cũng được khẳng định qua kết quả khảo sát của nhóm nghiên cứu về mức độ cần thiết đối với các đề xuất bổ sung vào văn bản hướng dẫn thực hiện bộ tiêu chuẩn đánh giá chất lượng CSGDĐH ban hành tại Thông tư 12 . Có 9/9 nội dung được đề xuất bổ sung đều được trên $86 \%$ số người trả lời cho rằng cần thiết thực hiện. Qua kết quả mô tả (Hình 1) có thể thấy, để thực hiện hiệu quả hơn công tác tự đánh giá, đánh giá ngoài CSGDĐH theo Thông tư 12 , các văn bản hướng dẫn thực hiện Bộ tiêu chuẩn này cần cung cấp thêm hệ thống văn bản pháp quy/các quy định liên quan để tham chiếu mức độ đáp ứng các yêu cầu của CSGDĐH theo nội hàm của tiêu chí; đồng thời cần có hướng dẫn chi tiết các đối tượng và nội dung cần quan sát trong quá trình đánh giá ngoài và đặc biệt là cần có phần mềm hỗ trợ tính toán các số liệu định lượng để hỗ trợ chuyên gia phân tích, đối sánh trong quá trình đánh giá ngoài (tỉ lệ đánh giá cần thiết đối với hai nội dung này đều đạt $89,4 \%$ ). Ngoài ra, có $86,5 \%$ số ý kiến cho rằng cần cung cấp các câu hỏi chẩn đoán để cụ thể hoá nội dung các mốc chuẩn của tiêu chí; $90,4 \%$ số người trả lời đề xuất cần cung cấp thêm các minh chứng tương ứng để có đầy đủ thông tin trả lời các câu hỏi chẩn đoán; 91,3\% số ý kiến trả lời yêu cầu rằng đối với từng tiêu chí cần thiết chỉ ra các tiêu chí liên quan khác trong Bộ tiêu chuẩn để hỗ trợ đối chiếu thông tin và xác định mức độ đạt yêu cầu tiêu chí. Kết quả này cũng cho thấy, cần thiết phải chi tiết hoá các công cụ để đo lường tiêu chí, giải quyết từng bước các vấn đề và yêu cầu trong thực tiễn đánh giá, kiểm định chất lượng các CSGDĐH như đã đề cập ở trên. 


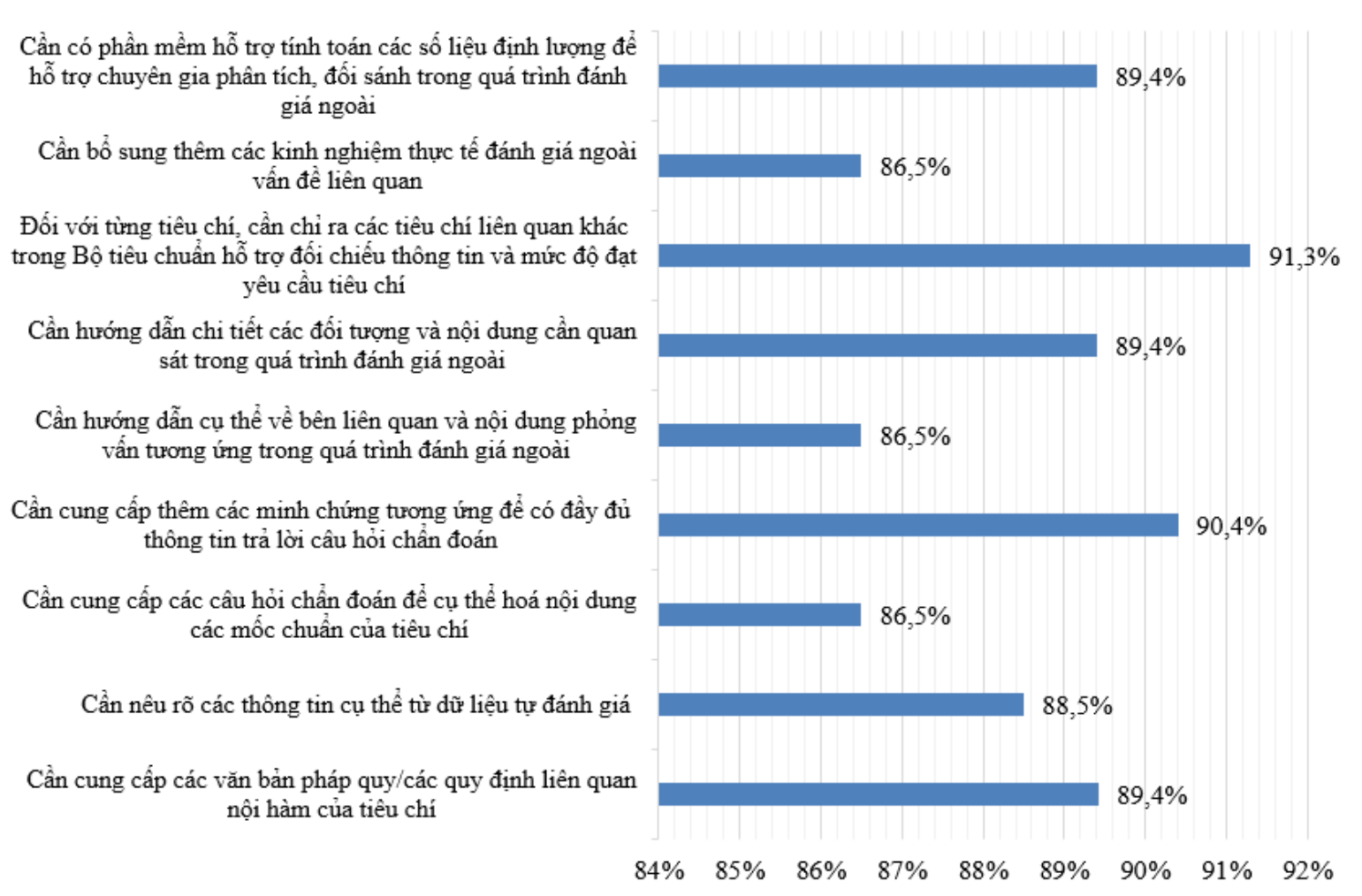

Hình 1 . Tỉ lệ người trả lời đánh giá là cần thiết (mức 4 và 5 ) đối với các đề xuất bổ sung trong văn bản hướng dẫn thực hiện Bộ tiêu chuẩn đánh giá chất lượng CSGDĐH $(N=104)$.

3.2. Quy trình xây dựng và Bộ công cu đo lường múc độ đảm bảo chất lương của co sơ giáo dục đại học

Như các nghiên cứu đã chỉ rõ, KĐCLGD dựa trên các tiêu chuẩn tối thiểu (cũng có thể gọi là "ngưỡng") để đánh giá và công nhận CSGDĐH đạt chuẩn chất lượng. Các bước thực hiện đánh giá gồm: "xem xét các minh chưng, thăm quan cơ sở vật chất và phỏng vấn các cán bộ giảng viên và hành chính, viết báo cáo đánh giá,..." [15]. Trong đánh giá, kiểm định chất lượng cơ sở giáo dục và các hoạt động đào tạo cần sử dụng các chỉ số (indicators) phù hợp đối với từng loại hoạt động. Có thể thiết kế các công cụ đánh giá hữu ích, có độ tin cậy để thực hiện đánh giá [16]. Đồng thời, xác định loại hình đánh giá, phương pháp đánh giá, mô hình đo lường, chỉ số độ tin cậy, thang đo, kỹ thuật định lượng, yếu tố (hoặc kích thước chất lượng) và các biến có khả năng bị ảnh hưởng bởi chúng [17]. Ở mỗi quốc gia, mỗi tổ chức KĐCLGD có những yêu cầu chuẩn chất lượng, cũng như cách thức, phương pháp đánh giá khác nhau, nhưng đều xác định rõ là kiểm định chất lượng cần phải kết hợp chặt chẽ với các công cụ khác để tạo hiệu quả và việc thu thập thông tin để đưa ra nhận định, đánh giá, quyết định mức đạt của tiêu chí là điều quan trọng tối ưu. Các phương pháp thu thập thông tin thường được sử dụng là nghiên cứu tài liệu, quan sát, phỏng vấn bán cấu trúc/phỏng vấn sâu/phỏng vấn nhóm tập trung, thảo luận nhóm, trắc nghiệm, thực nghiệm xã hội,... [18]. Đặc biệt, nền tảng công nghệ thông tin là công cụ hỗ trợ tích cực cho các đánh giá định lượng cũng như định tính.

Căn cứ các vấn đề lý luận và thực tiễn đã phân tích ở mục 3.2, nhóm nghiên cứu tiến hành xây dựng Bộ công cụ đo lường mức độ ĐBCL của CSGDĐH thông qua việc chi tiết 
hoá các yêu cầu đánh giá chất lượng của CSGDĐH theo hướng dẫn tại các công văn số 768 và số 1668 của Bộ GDĐT. Như đã nêu ở trên, nếu như quan điểm sử dụng của Bộ tiêu chuẩn đánh giá chất lượng CSGDĐH tại Thông tư 12 dùng để đánh giá, kiểm định chất lượng CSGDĐH và bộ tiêu chuẩn của $\mathrm{AUN}-\mathrm{QA}$ tiếp cận theo quan điểm đánh giá ĐBCL của CSGDĐH thì Bộ công cụ do nhóm nghiên cứu xây dựng sẽ được sử dụng để đo lường mức độ ĐBCL của CSGDĐH. Quan điểm của nghiên cứu này là: Việc xây dựng Bộ công cụ được tiếp cận theo quan điểm đánh giá mức độ ĐBCL của CSGDĐH, tham chiếu quan điểm đánh giá $Đ B C L$ của $A U N-Q A$ và tuân theo các nguyên tắc gồm: i) đảm bảo tính toàn diện - thu thập được đa dạng thông tin từ nhiều nguồn khác nhau; ii) đánh giá theo quy định và nguyên lý; iii) ứng dụng công nghệ thông tin trong đánh giá - kết nối tối đa các thông tin, dữ liệu và iv) đánh giá theo chuẩn/đối sánh.

Theo quan điểm này, nhóm nghiên cứu đã tiến hành xây dựng Bộ công cụ đo lường mức độ ĐBCL của CSGDĐH theo quy trình chặt chẽ, khoa học gồm 6 bước, được khái quát ở Hình 2.

Với mục đích hỗ trợ các CSGDĐH, các chuyên gia đánh giá thuộc các tổ chức KĐCLGD trong thực hiện tự đánh giá, đánh giá ngoài, nhóm nghiên cứu xác định các yêu cầu đối với Bộ công cụ̣, đảm bảo các nguyên tắc đánh giá đã đề cập ở trên. Theo đó, Bộ công cụ cần chi tiết hóa các yêu cầu đối với việc đánh giá mức độ ĐBCL của CSGDĐH theo Bảng hướng dẫn đánh giá kèm theo các công văn 768 và 1668 , đồng thời lượng hóa các yêu cầu này để hỗ trợ xác định chính xác hơn mức độ $\mathrm{DBCL}$ của CSGDĐH trong thực tiễn đánh giá. Bảng hướng dẫn đánh giá ngoài CSGDĐH theo Công văn 1668 sẽ được bổ sung 9 nội dung như các ý kiến góp ý từ phỏng vấn các chuyên gia và qua kết quả khảo sát bằng phiếu hỏi đối với các đối tượng liên quan trực tiếp đến công tác ĐBCL, KĐCLGD CSGDĐH; đảm bảo tính toàn diện trong đánh giá mỗi tiêu chí, tiêu chuẩn (như đã nêu ở mục 3.2). Những nội dung này cũng đảm bảo yêu cầu khoa học đối với phương pháp đánh giá đa chiều, có đủ thông tin tin cậy để đưa ra các nhận định đánh giá (bao gồm nghiên cứu hồ sơ, quan sát, phỏng vấn, phân tích dữ liệu, quan sát,...) [19]. Việc hướng dẫn thu thập thông tin sẽ căn cứ trên đa dạng nguồn, cả định lượng lẫn định tính, đảm bảo tính khách quan, tính thực tế và logic trong kết nối và phân tích thông tin, đánh giá 360 độ với cùng một vấn đề và khi xem xét tổng thể để đưa ra quyết định chính xác về mức độ ĐBCL của CSGDĐH. Trên cơ sở đó, mô hình Bộ công cụ được xây dựng; sau khi hoàn thiện được trình bày ở Hình 3.

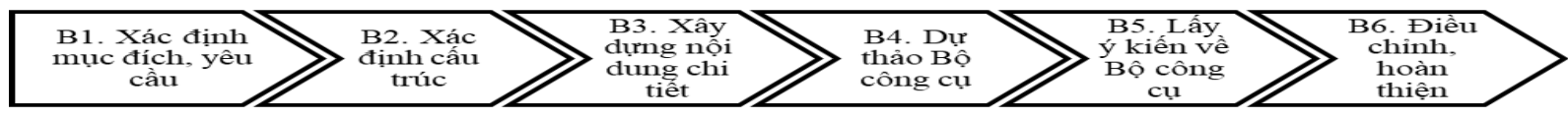

Hình 2. Quy trình xây dựng Bộ tiêu chuẩn đánh giá chất lượng CSGDĐH của nghiên cứu.

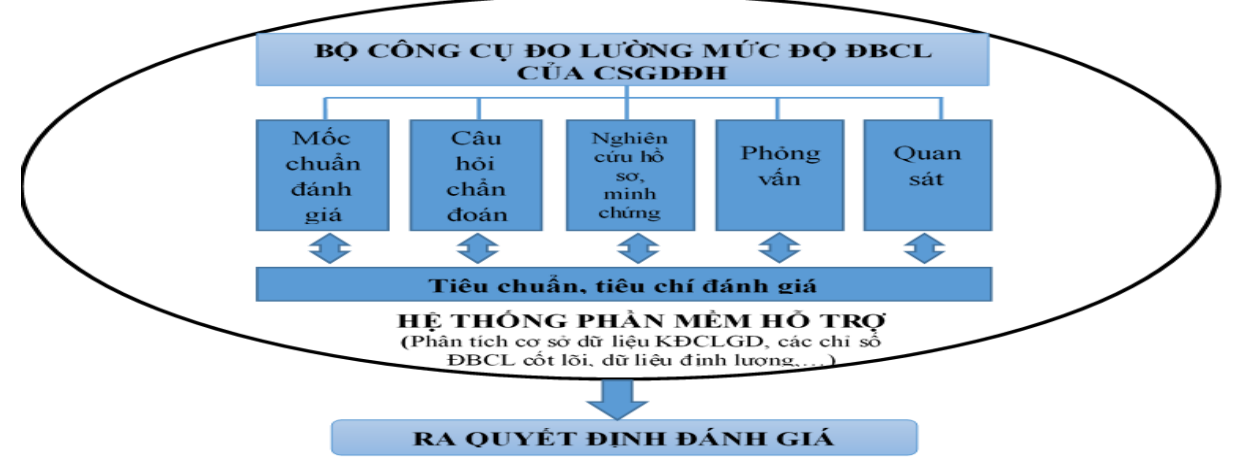

Hình 3. Mô hình Bộ công cụ đo lường mức độ ĐBCL của CSGDĐH. 
Theo mô hình này, cấu trúc của Bộ công cụ được xác định, phát triển dựa trên bảng hướng dẫn đánh giá ngoài CSGDĐH theo Công văn 1668 (trước đây là Công văn 768), đồng thời xây dựng thêm các nội dung hướng dẫn cụ thể đối với từng tiêu chí đánh giá căn cứ trên các luận cứ khoa học về phương pháp đo lường, đánh giá, kết quả khảo sát và tham vấn các chuyên gia. Các khái niệm được làm rõ và mỗi nội dung hướng dẫn đánh giá từng tiêu chí được xây dựng chi tiết, đảm bảo bám sát nội hàm tiêu chí. Trong khuôn khổ bài báo, nhóm nghiên cứu chỉ mô tả về các nội dung trong Bộ công cụ (Bảng 1).

Bản hướng dẫn đánh giá chi tiết 25 tiêu chuẩn sau khi hoàn thiện được tích hợp lại thành dự thảo Bộ công cụ đo lường mức độ ĐBCL của CSGDĐH. Nhóm nghiên cứu đã tiến hành rà soát, thảo luận thống nhất, điều chỉnh các nội dung đánh giá giữa các tiêu chí, tiêu chuẩn để đảm bảo sự thống nhất, chặt chẽ; sau đó gửi dự thảo tới các kiểm định viên, đánh giá viên, cán bộ quản lý và cán bộ $\mathrm{OBCL}$ tại các CSGDĐH để lấy ý kiến góp ý. Các ý kiến thu thập được từ các khảo sát, phỏng vấn và tham vấn chuyên gia được tập hợp, sử dụng các phần mềm chuyên dụng để xử lý và phân tích; kết quả được sử dụng làm căn cứ để điều chỉnh, hoàn thiện Bộ công cụ.

Quy trình trên cho thấy Bộ công cụ đã được xây dựng một cách bài bản, khoa học thông qua hệ thống các phương pháp thu thập thông tin đảm bảo đánh giá toàn diện, theo quy định và nguyên lý. Kết quả khảo sát (Bảng 2) cũng cho thấy có trên $80 \%$ ý kiến đồng ý đối với 12/13 nội dung được xác định trong Bộ công cụ, trong đó có 90,4\% số ý kiến đồng ý rằng Trình tư các nội dung huoóng dẫn trong Bộ công cu được sắp xếp logic, thống nhất. Trong các nội dung hướng dẫn của Bộ công cụ, nội dung các tiêu chí có nội hàm liên quan và các kinh nghiệm đánh giá ngoài có tỉ lệ khá cao người phản hồi $(86,5 \%)$ đánh giá phù hợp với thực tiễn. Tuy nhiên, vẫn còn một số ý kiến chưa đồng ý với các nhận định $C a ́ c$ văn bản pháp quy tham chiếu được phân loại rõ ràng, cập nhật (22,1\% người trả lời); Thông tin hố trọ đánh giá giúp xác định rõ mức độ đáp ứng yêu cầu của tiêu chí ( $20 \%$ số phản hồi) và Các minh chứng úng với câu hỏi chẩn đoán giúp tìm kiếm thông tin chẩn đoán mức độ đáp ứng yêu cầu tiêu chí (19,7\% số phản hồi). Ngoài ra, có một số ý kiến góp ý thu được từ phỏng vấn chuyên gia như: " $B \hat{o}$ công cu cần được đối sánh với các bộ công cu tuoong đương của các co quan quản lý nhà nước mà CSGDĐH phải trục tiếp báo cáo. Phải xác định đươc mưc đáp úng chung, dùng chung và tránh được chồng chéo văn bản báo cáo gây lãng phí và sai khác số liệu"; "Bộ công cu nên thiết kế thêm đánh giá chi tiết với tùng tiêu chuẩn để có cơ sở cải tiến bộ tiêu chuẩn tại Thông tu 12", hay "Nên xây dựng mức đáp úng cho tùng loại truờng cu thể (truoòng công/tu; truoòng nghiên cúu/nghiên cứu ưng dụng)",... là những lưu ý cần thiết để nhóm nghiên cứu tiếp tục rà soát, điều chỉnh và hoàn thiện Bộ công cụ (Bảng 2).

Bảng 1. Bảng hướng dẫn chi tiết đánh giá theo từng tiêu chí trong Bộ công cụ

\begin{tabular}{|c|c|}
\hline Nội dung & Mô tả \\
\hline Tiêu chí (theo Thông tư 12) & $\begin{array}{l}\text { Trình bày hệ thống tiêu chí đánh giá theo Bộ tiêu chuẩn đánh giá } \\
\text { chất lượng CSGDĐH theo Thông tư } 12 \text {. }\end{array}$ \\
\hline $\begin{array}{l}\text { Mô tả yêu cầu của tiêu chí theo Công văn } \\
1668\end{array}$ & $\begin{array}{l}\text { Trình bày các mô tả yêu cầu của tiêu chí theo hướng dẫn thực } \\
\text { hiện tại Công văn } 1668 \text {. }\end{array}$ \\
\hline $\begin{array}{l}\text { Mốc chuấn tham chiếu tối thiểu để đánh } \\
\text { giá tiêu chí đạt mức } 4 \text { theo Công } \\
\text { văn } 1668\end{array}$ & $\begin{array}{l}\text { Trình bày các mốc chuấn cụ thế hóa yêu cầu của tiêu chí, giúp } \\
\text { tham chiếu mức độ yêu cầu tối chiểu cần đạt của tiêu chí theo } \\
\text { hướng dẫn tại Công văn } 1668 \text {. }\end{array}$ \\
\hline $\begin{array}{l}\text { Gợi ý nguồn minh chứng theo Công văn } \\
1668\end{array}$ & $\begin{array}{l}\text { Trình bày hệ thống các minh chứng tối thiểu và mở rộng cần có, } \\
\text { hồ trợ khai thác thông tin xác định mức độ đạt yêu cầu của tiêu } \\
\text { chí trong quá trình đánh giá. }\end{array}$ \\
\hline
\end{tabular}




\begin{tabular}{|c|c|}
\hline Nội dung & Mô tả \\
\hline $\begin{array}{l}\text { Văn bản pháp quy của Nhà nước, Bộ } \\
\text { GDĐT, đơn vị chủ quản và văn bản của } \\
\text { nhà trường cần tham chiếu }\end{array}$ & $\begin{array}{l}\text { Trình bày hệ thống các văn bản pháp quy theo các cấp ban hành } \\
\text { và theo lĩnh vực liên quan, giúp dẫn chiếu thông tin để xác định } \\
\text { mức độ thực hiện của CSGDĐH đối với các quy định hiện hành } \\
\text { liên quan đến nội dung tiêu chí được đánh giá. }\end{array}$ \\
\hline $\begin{array}{l}\text { Thông tin hỗ trợ đánh giá (từ dữ liệu Báo } \\
\text { cáo Tự đánh giá và các bảng biểu do Nhà } \\
\text { trường và tồ chức KĐCLGD } \\
\text { cung cấp) }\end{array}$ & $\begin{array}{l}\text { Liệt kê cư thể (nội dung, vị trí) các thông tin từ dữ liệu định } \\
\text { lượng, định tính trong cơ sờ dữ liệu tự đánh giá của CSGDĐH, } \\
\text { các bảng biểu do Nhà trường, tô chức KĐCLGD cung cấp, giúp } \\
\text { người sử dụng xác định rõ mức độ đáp ứng yêu cầu của tiêu chí } \\
\text { được xem xét. }\end{array}$ \\
\hline $\begin{array}{l}\text { Các câu hỏi chẩn đoán ứng với mô tả tiêu } \\
\text { chí (thiết kế theo P-D-C-A) }\end{array}$ & $\begin{array}{l}\text { Trình bày hệ thống các câu hỏi được thiết kế theo chu trình P-D- } \\
\text { C-A (Plan-Do-Check-Act), tương ứng với các mốc chuẩn. Các } \\
\text { câu hỏi sẽ giúp chẩn đoán vấn đề của CSGDĐH về tiêu chí được } \\
\text { đánh giá, xác định xem CSGDĐH có đạt được mốc chuẩn tham } \\
\text { chiếu tối thiểu hay không và đáp ứng yêu cầu của tiêu chí ở mức } \\
\text { độ nào. }\end{array}$ \\
\hline $\begin{array}{l}\text { Tên các minh chứng ứng với phần trả lời } \\
\text { câu hỏi chẩn đoán (liệt kê tên, và nên có } \\
\text { file minh chứng kèm theo) }\end{array}$ & $\begin{array}{l}\text { Liệt kê cụ thế các tài liệu, dữ liệu chứa thông tin giúp trả lời câu } \\
\text { hỏi chẩn đoán; các tài liệu phù hợp thực tiễn, đảm bảo tương } \\
\text { thích với hệ thống minh chứng gợi ý (mục } 4 \text { ) và hỗ trợ làm rõ } \\
\text { mức độ đạt yêu cầu tiêu chí. }\end{array}$ \\
\hline $\begin{array}{l}\text { Phỏng vấn các bên liên quan (kiểm tra } \\
\text { các thông tin phục vụ trả lời cho câu hỏi } \\
\text { chẩn đoán) }\end{array}$ & $\begin{array}{l}\text { Trình bày các đối tượng và nội dung phỏng vấn tương ứng trong } \\
\text { quá trình đánh giá ngoài, giúp khai thác thông tin trả lời các câu } \\
\text { hỏi chẩn đoán được đưa ra hoặc để kiểm tra lại các thông tin } \\
\text { đã có. }\end{array}$ \\
\hline $\begin{array}{l}\text { Quan sát (kiểm tra các thông tin phục vụ } \\
\text { trả lời cho câu hỏi chẩn đoán) }\end{array}$ & $\begin{array}{l}\text { Trình bày các đối tượng cần quan sát trong quá trình đánh giá } \\
\text { ngoài, bồ sung thêm các thông tin trả lời câu hỏi chẩn đoán mà hồ } \\
\text { sơ, minh chứng hay việc phỏng vấn không khai thác được hoặc để } \\
\text { kiềm tra lại các thông tin đã có. }\end{array}$ \\
\hline $\begin{array}{l}\text { Tiêu chí liên quan (xem xét các tiêu chí } \\
\text { liên quan khác để rà soát thông tin và } \\
\text { đánh giá mức đạt tiêu chí) }\end{array}$ & $\begin{array}{l}\text { Liệt kê các tiêu chí khác trong Bộ tiêu chuấn có nội hàm liên } \\
\text { quan với tiêu chí đang được xem xét để rà soát thông tin và đánh } \\
\text { giá mức đạt tiêu chí. }\end{array}$ \\
\hline $\begin{array}{l}\text { Kinh nghiệm trong quá trình đánh } \\
\text { giá ngoài }\end{array}$ & $\begin{array}{l}\text { Trình bày các kinh nghiệm đánh giá ngoài, các thực hành tốt và } \\
\text { đối sánh được rút ra từ kinh nghiệm thực tiê̂n trong quá trình đánh } \\
\text { giá ngoài, đảm bảo phù hợp thực tiễn để hỗ trợ đưa ra quyết định } \\
\text { đánh giá. }\end{array}$ \\
\hline
\end{tabular}

Bảng 2. Kết quả lấy ý kiến cán bộ quản lý các cấp, chuyên gia thuộc tổ chức KĐCLGD, cán bộ quản lý và cán bộ ĐBCL giáo dục các $\mathrm{CSGDĐH,} \mathrm{về} \mathrm{Bộ} \mathrm{công} \mathrm{cụ} \mathrm{đo} \mathrm{lường} \mathrm{mức} \mathrm{độ} \mathrm{ĐBCL} \mathrm{của} \mathrm{CSGDĐH}$

\begin{tabular}{|c|l|c|}
\hline TT & \multicolumn{1}{|c|}{ Nội dung } & Tỉ lệ đồng ý \\
\hline 1 & $\begin{array}{l}\text { Các văn bản pháp quy tham chiếu giúp xác định rõ mức độ thực hiện các quy định } \\
\text { hiện hành liên quan đến nội dung tiêu chí }\end{array}$ & $82,7 \%$ \\
\hline 2 & Các văn bản pháp quy tham chiếu được phân loại rõ ràng, cập nhật & $77,9 \%$ \\
\hline 3 & Thông tin hỗ trợ đánh giá được nêu cụ thể, phù hợp với thực tiễn & $83,7 \%$ \\
\hline 4 & Thông tin hỗ trợ đánh giá giúp xác định rõ mức độ đáp ứng yêu cầu của tiêu chí & $80,8 \%$ \\
\hline 5 & Thứ tự các câu hỏi chẩn đoán tương ứng với mốc chuẩn tham chiếu & $81,7 \%$ \\
\hline 6 & Nội dung câu hỏi chẩn đoán giúp xác định mức độ đáp ứng yêu cầu của tiêu chí & $81,7 \%$ \\
\hline 7 & Các minh chứng được liệt kê cụ thể tương ứng với câu hỏi chẩn đoán, phù hợp với thực tiễn & $82,7 \%$ \\
\hline 8 & $\begin{array}{l}\text { Các minh chứng ứng với câu hỏi chẩn đoán giúp tìm kiếm thông tin chẩn đoán mức } \\
\text { độ đáp ứng yêu cầu tiêu chí }\end{array}$ & $80,8 \%$ \\
\hline
\end{tabular}




\begin{tabular}{|c|l|c|}
\hline TT & \multicolumn{1}{|c|}{ Nội dung } & Tỉ lệ đồng ý \\
\hline 9 & Các đối tượng và nội dung phỏng vấn tương ứng được xác định rõ ràng, đầy đủ & $81,7 \%$ \\
\hline 10 & Đối tượng quan sát được lựa chọn phù hợp & $82,7 \%$ \\
\hline 11 & Các tiêu chí có nội hàm liên quan được xác định phù hợp & $86,5 \%$ \\
\hline 12 & Các kinh nghiệm đánh giá ngoài phù hợp sử dụng trong thực tiễn & $86,5 \%$ \\
\hline 13 & Trình tự các nội dung hướng dẫn trong Bộ công cụ được sắp xếp logic, thống nhất & $90,4 \%$ \\
\hline
\end{tabular}

Với Bộ công cụ này, hệ thống câu hỏi chẩn đoán được xây dựng bám sát các mốc chuẩn tham chiếu của Bộ GDĐT, giúp làm rõ mức độ đáp ứng yêu cầu của tiêu chí. Cùng với đó, hệ thống hồ sơ, minh chứng bao gồm các văn bản pháp quy của Nhà nước, Bộ GDĐT, đơn vị chủ quản và văn bản của $\mathrm{CSGDĐH} \mathrm{mà} \mathrm{chuyên} \mathrm{gia}$ cần tham chiếu trong quá trình đánh giá; các minh chứng ứng với phần trả lời câu hỏi chẩn đoán để thu thập các thông tin trả lời câu hỏi chẩn đoán, giúp xác định mức độ đáp ứng yêu cầu của tiêu chí. Hướng dẫn phỏng vấn, quan sát giúp thu thập thêm các thông tin để trả lời câu hỏi chẩn đoán và khẳng định lại các thông tin đã thu được từ hồ sơ, minh chứng. Ngoài ra các thông tin có được từ việc tham chiếu các văn bản pháp luật, các tiêu chí khác liên quan trong Bộ tiêu chuẩn, kinh nghiệm thực tiễn đánh giá ngoài sẽ giúp thông tin được đối sánh, đảm bảo tính đa dạng và toàn diện. Cùng với hệ thống phần mềm hỗ trợ đánh giá, Bộ công cụ này sẽ hỗ trợ CSGDĐH cũng như các chuyên gia đánh giá ngoài đánh giá chuẩn xác hơn mức độ ĐBCL của CSGDĐH trước khi đưa ra quyết định đánh giá tổng thể.

Kết quả khảo sát của nhóm nghiên cứu cũng cho thấy: Có gần $90 \%$ người trả lời cho rằng Bộ công cụ sẽ hỗ trợ các $\mathrm{CSGDĐH} \mathrm{triển}$ khai hiệu quả các hoạt động ĐBCL $(89,9 \%)$ và phù hợp để các tổ chức KĐCLGD hướng dẫn triển khai các hoạt động đánh giá ngoài CSGDĐH (88,8\%). Bên cạnh đó, cũng có $82 \%$ ý kiến nhận định Bộ công cụ phù hợp để các kiểm định viên, đánh giá viên sử dụng để tự nâng cao năng lực đánh giá ngoài CSGDĐH. Ngoài ra cũng có $62,9 \%$ ý kiến cho rằng $\mathrm{B}$ ộ công cụ này sẽ hỗ trợ cơ quan quản lý nhà nước giám sát, đánh giá các hoạt động KĐCLGD.
Một số ý kiến khác đánh giá Bộ công cụ có thể hỗ trợ các giảng viên, nghiên cứu viên, người học trong quá trình nghiên cứu, tìm hiểu về lĩnh vực ĐBCL, KĐCLGD. Những kết quả này bước đầu cho thấy tính thực tiễn và hiệu quả của Bộ công cụ được xây dựng.

\section{Kiến nghị, đề xuất các giải pháp thực hiện}

Từ các kết quả nghiên cứu, có thể rút ra một số kiến nghị, đề xuất đối với các bên liên quan cụ thể như sau:

4.1. Bộ GDĐT nên định kỳ rà soát và đánh giá hiệu quả của hệ thống văn bản pháp lý liên quan đến tiêu chuẩn, quy trình KĐCLGD, trong đó có Thông tư 12 để cập nhật, điều chỉnh cho phù hợp và đáp ứng được yều cầu hướng dẫn các tổ chức KĐCLGD và các CSGDĐH thực thi trong thực tiễn; đồng thời, có thể tham khảo sử dụng Bộ công cụ đánh giá này để hỗ trợ cho công tác quản lý, giám sát và đánh giá việc thực hiện các hoạt động KĐCLGD.

4.2. Các CSGDĐH khi triển khai tự đánh giá theo Thông tư 12 nên chi tiết hoá các yêu cầu của các tiêu chí; có thể tham khảo bộ công cụ này để thiết lập ma trận nội dung và danh mục minh chứng cần có, đánh giá thực trạng và lập kế hoạch cải tiến chất lượng các hoạt động đáp ứng yêu cầu chuẩn chất lượng. Đồng thời, xây dựng hệ thống thông tin ĐBCL giáo dục, thực hiện đối sánh, phân tích thông tin một cách hữu ích để hỗ trợ các cấp ra quyết định và triển khai các hoạt động cải tiến phù hợp, đặc biệt chú trọng ứng dụng và khai thác hiệu quả công nghệ thông tin trong các hoạt động để vừa tiết kiệm được các nguồn lực vừa đạt được độ tin cậy, chính xác cho kết quả đánh giá.

4.3. Các tổ chức KĐCLGD có thể nghiên cứu áp dụng Bộ công cụ đánh giá này kết hợp 
với hệ thống phần mềm hỗ trợ đánh giá để công tác đánh giá được triển khai thuận lợi và đảm bảo tính chính xác, hiệu quả hơn cho kết quả đánh giá. Đồng thời, nên thường xuyên kết nối thông tin với các CSGDĐH để cập nhật cơ sở dữ liệu trên phần mềm để phục vụ cho đánh giá giữa kỳ và chu kỳ đánh giá tiếp theo.

4.4. Các kiểm định viên, đánh giá viên nên nghiên cứu kỹ các hướng dẫn cụ thể trong Bộ công cụ này để sử dụng trong các hoạt động đánh giá, đồng thời nắm bắt, sử dụng hiệu quả phần mềm hỗ trợ để đưa ra các thông tin và nhận định, đánh giá vừa đảm bảo các nguyên tắc, vừa chuẩn xác đối với thực trạng. Ngoài ra, cũng nên thường xuyên cập nhật kiến thức và chia sẻ kinh nghiệm, học hỏi các thực hành tốt để có thể đưa ra được những kiến nghị phù hợp, có tầm chiến lược nhằm giúp $\mathrm{CSGDĐH} \mathrm{cải}$ tiến, nâng cao chất lượng và hướng tới đạt được các mục tiêu chiến lược đã xác định.

\section{Kết luận}

Nhóm nghiên cứu đã tiến hành xây dựng Bộ công cụ đo lường mức độ $\mathrm{ĐBCL}$ của $\mathrm{CSGDĐH}$ căn cứ hướng dẫn đánh giá ngoài $\mathrm{CSGDĐH}$ theo Thông tư 12 của Bộ GDĐT, tham chiếu quan điểm đánh giá $\mathrm{DBCL}$ của $\mathrm{AUN}-\mathrm{QA}$ và tuân theo nguyên tắc đánh giá toàn diện, đảm bảo đánh giá theo nguyên tắc và nguyên lý, kết hợp ứng dụng công nghệ thông tin để xây dựng cơ sở dữ liệu đối sánh và đánh giá. Bài viết đã trình bày quy trình xây dựng và chi tiết hoá công cụ đo lường mức độ ĐBCL của CSGDĐH để sử dụng trong quá trình tự đánh giá, quá trình đánh giá ngoài theo Bộ tiêu chuẩn đánh giá chất lượng CSGDĐH do Bộ GDĐT ban hành. Đồng thời, qua đó đề xuất một số giải pháp khi sử dụng Bộ công cụ trong các hoạt động ĐBCL bên trong CSGDĐH, hỗ trợ các chuyên gia đánh giá ngoài và các tổ chức KĐCLGD đưa ra quyết định đánh giá. Bộ công cụ sử dụng đa dạng các phương pháp thu thập thông tin, kết hợp với phần mềm hỗ trợ sẽ là công cụ hiệu quả giúp các CSGDĐH, các chuyên gia đánh giá ngoài, tổ chức KĐCLGD đánh giá, xác định chính xác mức độ ĐBCL của các CSGDĐH để có các cải tiến chất lượng kịp thời, phù hợp. Đồng thời giúp các cơ quan quản lý Nhà nước giám sát, đánh giá hiệu quả việc triển khai KĐCLGD đại học.

\section{Lời cảm ơn}

Công trình nghiên cứu này được tài trợ bởi Đại học Quốc gia Hà Nội với Đề tài: Nghiên cứu xây dựng bộ công cụ và phần mềm đánh giá mức độ ĐBCL giáo dục, mã số QG.19.62.

\section{Tài liệu tham khảo}

[1] National Assembly of Vietnam, Law on amendments to the Law on Higher Education, Law No. 34/2018/QH14, issued on 19 November 2018.

[2] Ministry of Education and Training, Circular No. 12/2017/TT-BGDĐT promulgating the Regulations on the quality accreditation of higher education institutions, issued on 19 May 2017.

[3] Quality Control Department, Ministry of Education and Training, Documentary No. 766/QLCL-KĐCLGD on guiding self-assessment of quality of higher education institutions, issued on 20 April 2018.

[4] Quality Control Department, Ministry of Education and Training, Documentary No. 767/QLCL-KĐCLGD on guiding external evaluation of higher education institutions, issued on 20 April 2018.

[5] Quality Control Department, Ministry of Education and Training, Documentary No. 768/QLCL-KĐCLGD on the guidance of higher education institutions quality assessment standards, issued on 20 April 2018.

[6] Quality Control Department, Ministry of Education and Training, Official Dispatch No. 1668/QLCL-KĐCLGD on the replacement of the Evaluation Guideline issued together with Documentary No. 768/QLCL-KĐCLGD, issued on 20 April 2018.

[7] Ministry of Education and Training, List of educational institutions recognized as meeting educational quality standards. Retrieved from https://moet.gov.vn/giaoducquocdan/khao-thi-vakiem-dinh-chat-luong-giaoduc/Pages/Default.aspx?ItemID=6907 (accessed on 31 August 2020).

[8] Prime Minister of Government of the Socialist Republic of Vietnam, Decision approving the "Planning on the network of universities and colleges in the 2001-2010 period", issued on April 04, 2001. 
[9] Ministry of Education and Training, Decision No. 38/2004/QĐ-BGDĐT on issuing temporary regualtions on university quality assessment, issued on December 2, 2004.

[10] Central Committee of the Communist Party of Vietnam, Resolution No. 29-NQ/TW on "Fundamental and comprehensive innovation in education, serving industrialization and modernization in a socialist-oriented market economy during international integration" ratified in the 8th session, issued on November 4, 2013.

[11] National Assembly of Vietnam, Law on Higher Education, Law No. 08/2012/QH13, issued on June 18, 2012.

[12] Ministry of Education and Training, Directive No. 2119/CT-BGDĐT dated August 10, 2018 on Tasks and solutions for the 2018-2019 school year, Directive No. 2268/CT-BGDĐT dated August 8, 2019 on Tasks and solutions for the 2019-2020 school year and Directive No. 666 /CT-BGDĐT dated August 24, 2020 on Tasks and solutions for the 2020-2021 school year of the education sector, 2017.

[13] Asian University Network Quality Assurance, Guide to AUN-QA Assessment at Institutional Level, Version 2.0 (Vietnamese version), Vietnam National University Press, Hanoi, 2016.
[14] Ministry of Education and Training, Plan No. 118/KH-BGDĐT dated October 23, 2017 on Deploying the quality acreditation activities for the universities, pedagogy colleges and schools 2017, 2017.

[15] Vlãsceanu, Grünberg, and Pãrlea - Source: Training Materials - UNESCO, 2010.

[16] UNESCO, Internal Quality Assurance: Enhancing higher education quality and graduate employability, International Institute for Educational Planning Press, France, 2018.

[17] Jorge Pérez Rave, Leandro Muñoz Giraldo, What can't be ignored in service quality evaluation: Application contexts, tools and factors, 2014.

[18] Fernando Doménech Betoret \& Adela Descals Tomás, Evaluation of the University Teaching/Learning Process for the Improvement of Quality in Higher Education, 2010, p.165-178. https://www.tandfonline.com/doi/abs/10.1080/0 2602930301672.

[19] Division of Research, Evaluation and Communication, National Science Foundation, The 2002 User-Friendly Handbook for Project Evaluation, 2020. 